\title{
Visceral Obesity-Related Indices in the Identification of Individuals with Metabolic Syndrome Among Different Ethnicities in Xinjiang, China
}

This article was published in the following Dove Press journal: Diabetes, Metabolic Syndrome and Obesity: Targets and Therapy

\author{
Xianghui Zhang ${ }^{1, *}$ \\ Yusong Ding',* \\ Yinbao Shao' \\ Jia $\mathrm{He}^{\mathrm{l}}$ \\ Jiaolong $\mathrm{Ma}^{\prime}$ \\ Heng Guo' \\ Mulatibieke Keerman' \\ Jiaming Liu' \\ Huili $\mathrm{Si}^{2}$ \\ Shuxia Guo (D) ${ }^{1,3}$ \\ Rulin $\mathrm{Ma}^{\mathrm{I}}$ \\ 'Department of Public Health, Shihezi \\ University School of Medicine, Shihezi, \\ Xinjiang, People's Republic of China; \\ ${ }^{2}$ Department of Neurology, Shihezi People's \\ Hospital, Shihezi, Xinjiang, People's Republic \\ of China; ${ }^{3}$ Department of Pathology and Key \\ Laboratory of Xinjiang Endemic and Ethnic \\ Diseases (Ministry of Education), Shihezi \\ University School of Medicine, Shihezi, \\ Xinjiang, People's Republic of China \\ *These authors contributed equally to this \\ work
}

Correspondence: Shuxia Guo Department of Public Health, Shihezi University School of Medicine, Suite 72I, Building No. I, Beier Road, Shihezi, 832000, Xinjiang, People's Republic of China

Tel +86 I800-9932-625

Fax +86 993-2057-I53

Email gsxshzu@sina.com

Rulin Ma

Department of Public Health, Shihezi University School of Medicine, Suite 816, Building No. I, Beier Road, Shihezi, 832000,

Xinjiang, People's Republic of China

Tel +86 |330-9930-56|

Fax +86 993-2057-I53

Email marulin@shzu.edu.cn
Background: Few studies have focused on the predictive ability of visceral obesity-related indices for metabolic syndrome (MetS), especially in different ethnic groups. This study aimed to evaluate the applicability of visceral obesity-related indices for MetS screening among three major ethnic groups living in remote rural areas of Xinjiang.

Methods: Based on multistage stratified cluster random sampling method, 3,192 Uyghurs, 3,054 Kazakhs, and 3,658 Hans were recruited from Xinjiang, China. The Joint Interim Statement (JIS) criteria were used to define MetS. The receiver operating characteristic curve (ROC), area under the ROC curve (AUC), and predictive value of each visceral obesityrelated index were used to evaluate the predictive ability of MetS.

Results: After adjusting for potential confounding factors, the lipid accumulation product (LAP), Chinese visceral adiposity index (CVAI), waist-to-height ratio (WHtR), and atherogenic index of plasma (AIP) were significantly correlated with MetS for each ethnic group, and the odds ratios (ORs) for MetS increased across quartiles. LAP was best able to identify MetS status in Kazakhs (AUC=0.853) and Uyghurs (AUC=0.851), with optimal cut-offs being 36.3 and 28.2, respectively. Both LAP (AUC=0.798) and CVAI (AUC=0.791) most accurately identified MetS status in Hans, with the optimal cut-offs being 27.3 and 85.0, respectively. Moreover, the AUC of the combination of these visceral obesity-related indices is higher for each ethnic group. However, compared with LAP, the improved value of combined screening was not significant.

Conclusion: LAP had the best discriminative capability for the screening of MetS among Kazakhs, Uyghurs, and Hans. The screening ability of CVAI for MetS was similar to that of LAP in Hans. Thus, LAP may be a complementary indicator for assessing MetS in various ethnic groups.

Keywords: metabolic syndrome, visceral obesity-related indices, screening, ethnic groups

\section{Introduction}

Metabolic syndrome (MetS) is becoming a major health concern in both developing and developed countries. With an increasing prevalence worldwide, ${ }^{1}$ it is a major risk factor for type 2 diabetes (T2DM) and morbidity and mortality from cardiovascular disease (CVD). ${ }^{2}$ Recently, the diagnostic criteria of MetS are non-stringent. For example, for women, the cut-off value of high-density lipoprotein cholesterol (HDL-C) has changed from $<1.1 \mathrm{mmol} / \mathrm{L}$ in 2005 International Diabetes Federation (IDF) criteria $^{3}$ to $<1.3 \mathrm{mmol} / \mathrm{L}$ in the 2009 Joint Interim Statement (JIS) criteria, ${ }^{4}$ whose purpose is to identify more patients at risk and 
facilitate primary prevention. Hence, early identification and treatment of individuals at high risk of MetS is imperative to prevent the adverse consequences related to its development. ${ }^{5}$ Unfortunately, it is still a challenge to identify individuals with MetS early due to the unobvious physical discomfort and complex diagnostic criteria associated with this disorder.

Recently, numerous studies reported that visceral obesity, compared to overall obesity, might be a better determinant of metabolic diseases, dyslipidemia, MetS, and CVD. ${ }^{6,7}$ It is particularly essential in Chinese individuals, who generally tend to have more visceral fat but lower body and subcutaneous fat than those in Western populations. ${ }^{8}$ Thus, early and accurate diagnosis of visceral obesity might be of crucial significance for the prevention of MetS. Although Magnetic Resonance Imaging (MRI) and Computed Tomography (CT) are gold standards to determine visceral fat, these are impractical to use for large-scale screening in underdeveloped areas. Therefore, several surrogate visceral obesity-related indices have been developed. One of the most well-accepted and widely used of these indices is the waist-to-height ratio (WHtR), which approximately reflects $52-81 \%$ of visceral fat. ${ }^{9}$ However, WHtR cannot distinguish between abdominal subcutaneous and visceral adipose tissue, and can not effectively improve the determination of visceral fat beyond using waist circumference (WC) alone. ${ }^{10}$ The lipid accumulation product (LAP), an index based on the combination of WC and serum triglycerides (TG), was proposed as a reliable marker of central lipid accumulation, and was more effective in identifying CVD risk compared to body mass index (BMI). ${ }^{11}$ Indeed, LAP has been reported as a useful index for detecting insulin resistance, non-alcoholic fatty liver, and MetS in various studies. ${ }^{12-14}$ Based on the differences in body fat distribution between the Chinese population and Caucasians, ${ }^{15}$ a new index called the Chinese visceral adiposity index (CVAI) was developed. This index combines age, BMI, WC, TG, and HDL-C, and was found to be a reliable indicator of visceral fat dysfunction. ${ }^{16}$ Further, the atherogenic index of plasma (AIP), a composite index based on TG and HDL-C, ${ }^{17}$ has been used as a reference to estimate abdominal obesity ${ }^{18}$ and is commonly used as an optimal indicator of the risks for atherosclerosis and coronary heart disease. ${ }^{19,20}$ However, no studies have systematically compared the value of these visceral obesity-related indicators (WHtR, LAP, CVAI, and AIP) for the screening of MetS. More importantly, pronounced differences in body fat distribution exist among various ethnicities. ${ }^{21}$ Nevertheless, to date, few studies have focused directly on different ethnic populations.
Xinjiang is a multi-ethnic region of China (containing 47 ethnic groups). Among them, the Kazakhs, Uyghurs, and Hans account for $92.4 \%$ of Xinjiang's total population. In addition, most remote areas of Xinjiang are economically less developed compared with the remaining parts of China. ${ }^{22}$ Therefore, practical and effective indicators are urgently needed to screen MetS and other CVD risks. Thus, this multi-ethnic study was conducted to evaluate the feasibility of visceral obesity-related indices (ie, WHtR, LAP, CVAI, and AIP) for MetS screening among three ethnic populations (Kazakhs, Uyghurs, and Hans) living in rural areas of Xinjiang. Additionally, the present study also explored the optimal cut-offs for each indicator in different ethnic groups. The findings may provide a basis for the establishment and improvement of MetS screening and diagnostic strategies in Xinjiang and other areas of China.

\section{Methods}

\section{Study Setting and Population}

This Multi-ethnic Region Study was started in 2009 in Yili (Kazakhs), Shihezi city (Hans), Kashgar (Uyghurs), and Tumxuk (Uyghurs) of Xinjiang, which are nearly 2,800 miles away from Capital Beijing (As far as from the east coast to the west border of China). Multistage stratified random sampling method (prefecture-county-town-village) was adopted to recruit participants. First, Xinjiang was divided into South and North areas bound by Tianshan Mountain. Second, two representative prefectures were selected from the South (Kashgar and Tumxuk) and North (Shihezi and Yili), respectively, based on ethnicity, demographics, as well as economic and cultural status. Third, one county was randomly selected from each prefecture, and one town was randomly chosen in each county. Then, the corresponding villages in each town were selected using a stratified random sampling method. Finally, the local residents (aged $\geq 18$ years) who had been living in the village for at least 6 months were randomly chosen and interviewed.

The exclusion criteria of the study subjects are as follows: (1) Those who can not complete the blood sample collection, questionnaire survey, physical examination, or blood pressure measurement, (2) People with disabilities or mental disorders, (3) People with serious illness, (4) Pregnant women, (5) Obesity caused by disease, (6) Obesity caused by taking certain drugs, (7) Those who have not signed the informed consent form. 
A total of 11,419 participants were included in our analysis, with 1,390 and 125 individuals excluded due to missing laboratory and anthropometric data, respectively. The final analysis included 3,192 Kazakhs (mean age 49.8 years, male sex 42.1\%), 3,054 Uyghurs (mean age 42.5 years, male sex 50.8\%), and 3,658 Hans (mean age 44.3 years, male sex 39.4\%) (Figure 1).

\section{Assessment of MetS}

The measurement of WC, systolic blood pressure (SBP), and diastolic blood pressure (DBP) were performed by health professionals following a standard protocol. ${ }^{23}$ After an overnight fast, blood samples were collected in the morning. All samples were delivered to a clinical laboratory for further analysis. Fasting blood glucose (FBG), plasma TG and HDL-C concentrations were measured using a biochemical auto-analyzer (Olympus $\mathrm{AU}$ 2700; Olympus Diagnostics, Hamburg, Germany). MetS was defined according to the joint interim statement of the IDF Task Force (JIS criteria), with recommended WC cutoffs for the Chinese population. ${ }^{4}$

\section{Assessment of Visceral Obesity-Related Indices}

The visceral obesity-related indices mentioned in the current study, including WHtR, LAP, CVAI and AIP, were calculated using the following equations:

- WHtR $=$ WC $(\mathrm{cm}) /$ height $(\mathrm{cm})$

- LAP $($ men $)=(\mathrm{WC}(\mathrm{cm})-65) \times \mathrm{TG}(\mathrm{mmol} / \mathrm{L})$, or

$$
\begin{aligned}
& \text { LAP }(\text { women })=(\mathrm{WC}(\mathrm{cm})-58) \times \mathrm{TG}(\mathrm{mmol} / \mathrm{L})^{11} \\
& - \text { CVAI }(\text { men })=-267.93+0.68 * \text { age }+0.03 * \mathrm{BMI}+4.00 \\
& * \text { WC }+22.00 * \log 10 \mathrm{TG}-16.32 * \mathrm{HDL}-\mathrm{C}, \text { or } \\
& \text { CVAI }(\text { women })=-187.32+1.71 * \text { age }+4.23 * \mathrm{BMI} \\
& +1.12 * \mathrm{WC}+39.76 * \log 10 \mathrm{TG}-11.66 * \mathrm{HDL}-\mathrm{C}{ }^{16} \\
& -\mathrm{AIP}=\log (\mathrm{TG} / \mathrm{HDL})^{24}
\end{aligned}
$$

\section{Assessment of Other Covariates}

The personal data of the participants were collected using structured questionnaires administered through face-toface interviews. The questionnaire mainly inquired on demographics (age, sex, occupation, marriage status, etc.), lifestyle (eg, smoking status, alcohol consumption), and socioeconomic, dietary, and medical history.

\section{Statistical Analysis}

Continuous variables were described as means and standard deviations and were compared using Student's t-tests. Categorical data were presented as frequencies and were analyzed with the Chi-square tests. Multinomial logistic regression was performed to determine the association between the visceral obesity-related indices and the risk of MetS. Each visceral obesity-related index was divided into increasing quartile groups to calculate the odds ratio (OR) and 95\% confidence interval (CI) for MetS. To visualize the relationship between visceral obesity-related indices and the risk of MetS, the indicators of interest were also modeled as continuous variables using nomograms.

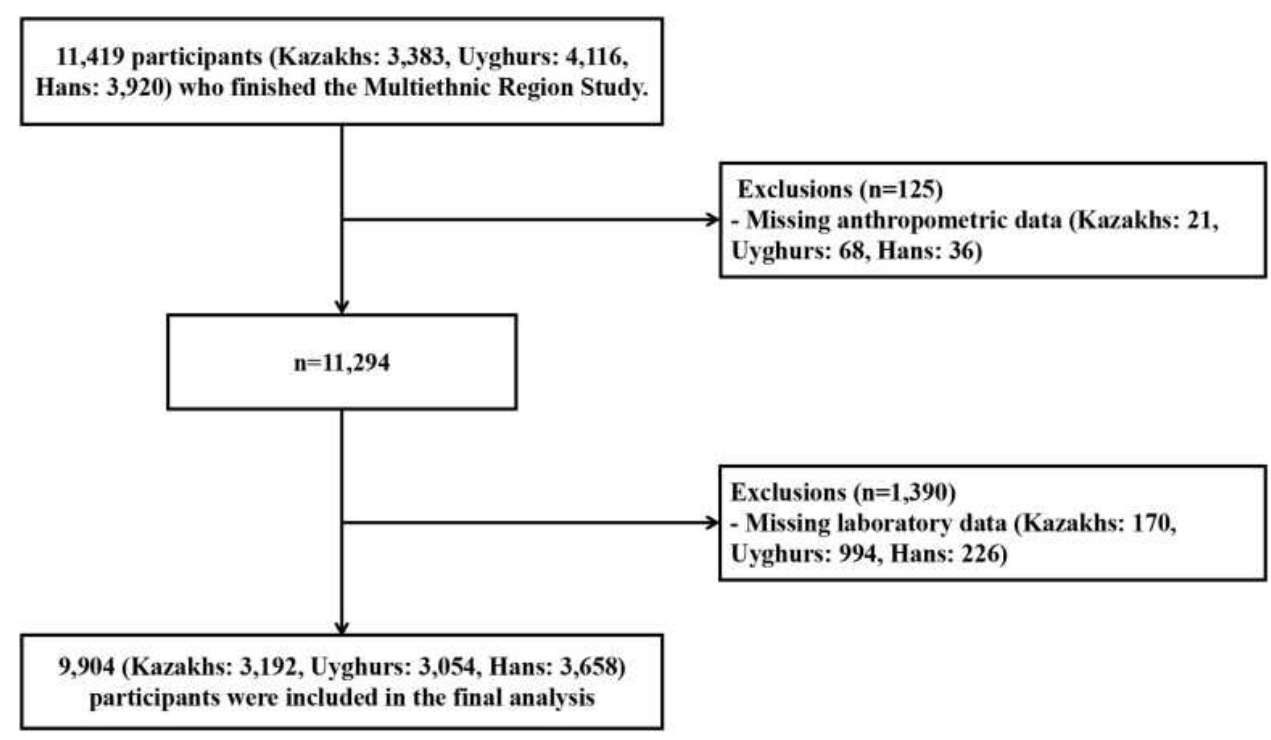

Figure I Flow chart of the multi-ethnic region study participants.

Note: All analyses presented in this paper were based on 9,904 surveyed participants, each with complete anthropometric, laboratory and covariable data. 
ROC analysis was applied to compare the ability of each indicator to screen for MetS, and the ROC curves were plotted for each of the indicators in Kazakhs, Uyghurs, and Hans, respectively. The overall screening accuracy was quantified using the AUC. An AUC of 0.5, 0.6 to $<0.7,0.7$ to $<0.8,0.8$ to $<0.9$, and $\geq 0.9$ corresponded to no discrimination, poor, acceptable, excellent, and outstanding discrimination, respectively. ${ }^{25}$ To reduce variance, a bootstrapping procedure was used 2000 times to validate the best AUC values and $95 \%$ CIs for MetS. ${ }^{26}$ Moreover, the comparisons of AUC values were performed using the method of DeLong et $\mathrm{al}^{27}$ and the optimal cut-off value was identified using the maximum value of Youden's index, which is calculated by sensitivity (Sen) plus specificity (Spe) minus 1 .

Statistical analyses were performed using SPSS version 23.0 (IBM Corp., Armonk, NY, USA), R software version 3.6.1 (https://www.r-project.org/, The R Foundation), and MedCalc statistical software version 15.2.2 (MedCalc Software bvba, Ostend, Belgium). Differences were considered statistically significant at a P-value $<0.05$ (twotailed).

\section{Results}

\section{Basic Characteristics of Study Participants}

A total of 3,192 Kazakhs (32.2\%), 3,054 Uyghurs (30.8\%), and 3,658 Hans (36.9\%) were analyzed in this study. For each ethnic population, the MetS group showed significantly higher values for age, weight, WC, BMI, SBP, DBP, TG, FBG, LAP, CVAI, AIP, and WHtR, and lower values for HDL-C and height than those in the NonMetS group (except for the height in Kazakhs, $p=0.914$ ) (Table 1).

\section{Associations of Visceral Obesity-Related Indices with MetS}

LAP, CVAI, AIP, and WHtR were strongly associated with MetS in three ethnicities after adjustment for potential confounders (age, sex, smoking and drinking habits, occupation, marital status, education, and BMI.) ( $\mathrm{p}$ for trend $<0.001$ ) (Figure 2). For all variables of interest in Kazakhs and Uyghurs, the LAP indicated the highest OR value for MetS (3.751 for Kazakhs and 3.726 for Uyghurs), followed by CVAI (3.002 for Kazakhs and 3.042 for Uyghurs), AIP (2.597 for Kazakhs and 2.863 for Uyghurs) and WHtR (2.154 for Kazakhs and 2.225 for Uyghurs). On the other hand, the highest OR in Hans was for CVAI, followed by LAP, AIP, and WHtR (OR=2.672, $2.571,2.229$, and 2.162 , respectively).

\section{Using the Nomogram}

For example, in Kazakhs, if the LAP level was 35, the point of 30 can be obtained from the points bar. Other variables can also be handled similarly. Presuming that the CVAI level of 60 had a point of 10, the AIP level of 0.2 was 70 points, and the WHtR level of 0.55 was 30 points. Then, the total score was $140(30+10+70+30)$. Finally, the risk of MetS was about $40 \%$ after marking a straight line from the total points to the risk axis (Figure 3).

\section{The ROC Analyses of MetS Screening}

In Kazakhs, the LAP had the largest AUC value of 0.853 (95\% CI: 0.840-0.865) among the four visceral obesityrelated indices. The optimal cut-off point was 36.3 based on the maximum Youden's index of 0.555 (Sen $=76.6 \%$, Spe $=78.9 \%$ ). In Uyghurs, the AUC values of LAP, CVAI, and AIP were all larger than 0.800 . The LAP had the highest discriminative ability for MetS (AUC $=0.851$, 95\% CI: 0.837-0.863), with the optimal cut-off point at 28.2 according to the maximum Youden's index of 0.561 (Sen $=78.6 \%$, Spe $=77.5 \%)$. The largest AUC for screening MetS in Hans was still observed for LAP (AUC = 0.798 , Youden's index $=0.454$, Sen $=73.9 \%$, Spe $=71.6 \%$ and optimal cut-off $=27.3)$ followed by CVAI (AUC $=$ 0.791 , Youden's index $=0.463$, Sen $=75.0 \%$, Spe $=71.3 \%$ and optimal cut-off $=85.0$ ). The ability of LAP and CVAI in screening for MetS were similar. In addition, for each ethnicity, when the four visceral obesity-related indices were combined, the diagnostic value for MetS was higher than that when taking the indices separately. Significant differences were detected between AUC values for all pairs of indices, except for the pair of LAP and CVAI in Hans. (Table 2 and Figure 4).

\section{Discussion}

The present study showed that visceral obesity-related indices such as LAP and CVAI are accurate indicators in identifying MetS, with LAP being the index with the strongest diagnostic accuracy for MetS among all the three ethnic populations. This is the first large-scale population-based study to systematically evaluate the screening value of four visceral obesity-indices (LAP, CVAI, LAP, and $\mathrm{WHtR}$ ) for identifying MetS in three ethnic populations in remote rural regions of China. The present study strengthened newly-found evidence that LAP is a simple 


\begin{tabular}{|c|c|c|c|c|c|c|c|c|c|c|c|c|c|c|c|c|c|}
\hline \multirow{4}{*}{ 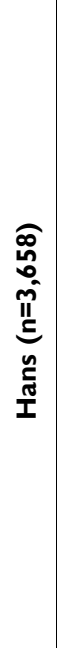 } & 吾 & 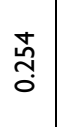 & $\begin{array}{l}\overline{8} \\
\dot{0} \\
\mathrm{v}\end{array}$ & $\frac{m}{0}$ & $\begin{array}{l}\bar{\delta} \\
\dot{0} \\
\dot{v}\end{array}$ & $\begin{array}{l}\overline{8} \\
\dot{0} \\
\mathrm{v}\end{array}$ & $\begin{array}{l}\overline{8} \\
\dot{i} \\
\mathrm{v}\end{array}$ & $\begin{array}{l}\bar{\delta} \\
\dot{0} \\
\dot{v}\end{array}$ & \begin{tabular}{|l}
$\bar{\delta}$ \\
$\overline{0}$ \\
$\mathrm{v}$
\end{tabular} & $\begin{array}{l}\bar{\delta} \\
\dot{v} \\
\mathrm{v}\end{array}$ & $\begin{array}{l}\overline{8} \\
\dot{0} \\
\mathrm{v}\end{array}$ & $\begin{array}{l}\bar{\delta} \\
\dot{0} \\
\mathrm{v}\end{array}$ & $\begin{array}{l}\overline{8} \\
\dot{0} \\
\mathrm{v}\end{array}$ & $\begin{array}{l}\bar{\delta} \\
\dot{0} \\
\mathrm{v}\end{array}$ & $\begin{array}{l}\overline{\mathrm{o}} \\
\overline{\mathrm{v}}\end{array}$ & $\begin{array}{l}\overline{8} \\
\dot{0} \\
\mathrm{v}\end{array}$ & \\
\hline & $\widetilde{\mathfrak{x}}$ & 惫 & 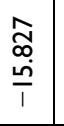 & $\underset{\substack{\mathfrak{v} \\
\mathfrak{i}}}{ }$ & $\begin{array}{l}\bar{m} \\
\stackrel{0}{\Lambda} \\
\end{array}$ & $\begin{array}{l}\bar{\sigma} \\
\dot{i} \\
\dot{i}\end{array}$ & 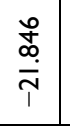 & $\begin{array}{l} \pm \\
\stackrel{0}{0} \\
\stackrel{i}{i} \\
i\end{array}$ & 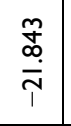 & 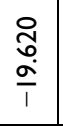 & $\begin{array}{c}\underset{\sim}{\sim} \\
\underset{\infty}{0} \\
\infty\end{array}$ & $\begin{array}{l}\sim \\
\stackrel{\sim}{0} \\
\stackrel{i}{T}\end{array}$ & 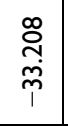 & 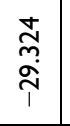 & $\begin{array}{c}\underset{c}{\infty} \\
\stackrel{\alpha}{\sigma} \\
\frac{\sigma}{1}\end{array}$ & 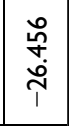 & \\
\hline & 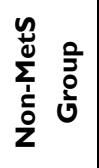 & 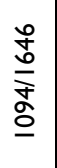 & $\begin{array}{c}\stackrel{0}{\dot{m}} \\
\stackrel{+}{+1} \\
\stackrel{j}{j}\end{array}$ & $\begin{array}{l}\tilde{S} \\
\infty \\
0 \\
+1 \\
0 \\
\underline{\underline{0}}\end{array}$ & 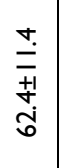 & $\begin{array}{c}\hat{0} \\
\stackrel{+}{+1} \\
\dot{d} \\
\infty\end{array}$ & 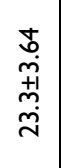 & 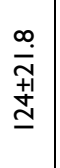 & 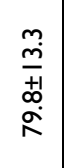 & 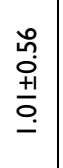 & 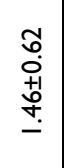 & $\begin{array}{c}\infty \\
0 \\
0 \\
0 \\
\infty \\
\stackrel{+}{+} \\
+\end{array}$ & 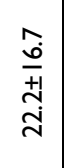 & $\begin{array}{l}0 \\
+ \\
+ \\
0 \\
0 \\
\dot{j} \\
0\end{array}$ & 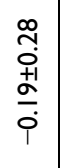 & $\left|\begin{array}{c}0 \\
0 \\
i+1 \\
\underline{+1} \\
0\end{array}\right|$ & \\
\hline & 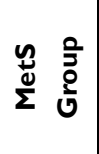 & $\begin{array}{c}\bar{n} \\
\stackrel{n}{\hat{f}} \\
\bar{m}\end{array}$ & 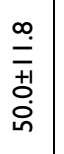 & $\begin{array}{l}\bar{\alpha} \\
o \infty \\
+1 \\
\mathbb{0} \\
\underline{1}\end{array}$ & $\frac{\stackrel{0}{m}}{\frac{\dot{m}}{\dot{p}}}$ & $\frac{m}{\frac{m}{m}}$ & 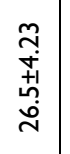 & 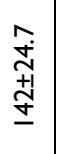 & 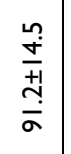 & 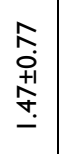 & $\begin{array}{l}n \\
n \\
0 \\
+1 \\
\\
\underline{-}\end{array} \mid$ & 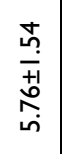 & 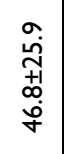 & $\begin{array}{l}\stackrel{\mathfrak{j}}{\mathfrak{J}} \\
\stackrel{+}{+} \\
\stackrel{\Xi}{=}\end{array}$ & 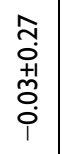 & $\begin{array}{l}0 \\
0 \\
0 \\
+1 \\
\hat{1} \\
0 \\
0\end{array}$ & \\
\hline \multirow{4}{*}{ 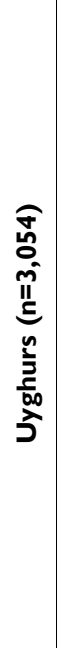 } & 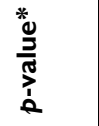 & $\overline{8}$ & $\begin{array}{l}\overline{8} \\
\dot{0} \\
\mathrm{v}\end{array}$ & o̊ & $\begin{array}{l}\bar{o} \\
\dot{0} \\
\mathrm{v}\end{array}$ & $\begin{array}{l}\overline{8} \\
\dot{0} \\
\mathrm{v}\end{array}$ & $\begin{array}{l}\overline{8} \\
\dot{0} \\
\mathrm{v}\end{array}$ & $\begin{array}{l}\overline{8} \\
\dot{0} \\
\dot{v}\end{array}$ & \begin{tabular}{|l}
$\bar{\delta}$ \\
$\overline{0}$ \\
$\mathrm{v}$
\end{tabular} & $\begin{array}{l}\bar{\delta} \\
\dot{0} \\
\mathrm{v}\end{array}$ & $\begin{array}{l}\overline{8} \\
\dot{0} \\
\mathrm{v}\end{array}$ & $\begin{array}{l}\overline{8} \\
\dot{0} \\
\mathrm{v}\end{array}$ & $\begin{array}{l}\overline{8} \\
\dot{0} \\
\mathrm{v}\end{array}$ & $\begin{array}{l}\overline{8} \\
\dot{0} \\
\mathrm{v}\end{array}$ & $\begin{array}{l}\overline{8} \\
\overline{0} \\
\mathrm{v}\end{array}$ & \begin{tabular}{|l}
$\overline{8}$ \\
$\dot{0}$ \\
$\mathrm{v}$
\end{tabular} & \\
\hline & $\widetilde{\widetilde{X}}$ & $\begin{array}{l}\stackrel{\circ}{0} \\
\stackrel{0}{0}\end{array}$ & $\begin{array}{c}\stackrel{\infty}{o} \\
\stackrel{m}{m} \\
\end{array}$ & $\stackrel{\sim}{\circ}$ & $\begin{array}{l}\stackrel{0}{\hat{N}} \\
\stackrel{0}{\circ}\end{array}$ & $\begin{array}{l}\frac{\hat{\infty}}{\mathbf{T}} \\
\hat{T}\end{array}$ & $\begin{array}{l}\stackrel{0}{6} \\
\stackrel{\leftrightarrow}{\mathfrak{i}} \\
1\end{array}$ & 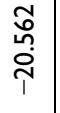 & $\begin{array}{c}\bar{ָ} \\
\stackrel{i}{T}\end{array}$ & $\begin{array}{l}\stackrel{\infty}{i} \\
\stackrel{i}{i} \\
\uparrow\end{array}$ & 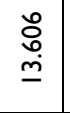 & $\begin{array}{l}\tilde{o} \\
\stackrel{\alpha}{\mathbf{i}} \\
i\end{array}$ & 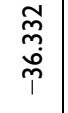 & 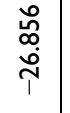 & $\frac{\underset{\sim}{\tilde{T}}}{\stackrel{T}{T}}$ & 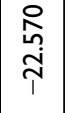 & \\
\hline & 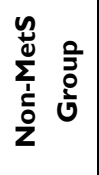 & 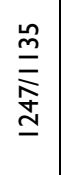 & $\begin{array}{l}\overline{6} \\
\frac{0}{+1} \\
\dot{+} \\
\dot{q}\end{array}$ & $\begin{array}{l}\text { o } \\
\infty \\
o \\
+1 \\
0 \\
\underline{0}\end{array}$ & $\begin{array}{l}\bar{n} \\
o \\
0+1 \\
0 \\
i \\
n\end{array}$ & 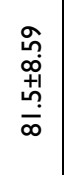 & $\begin{array}{l}\stackrel{8}{0} \\
\stackrel{+}{+} \\
\stackrel{N}{N}\end{array}$ & 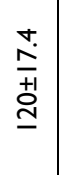 & 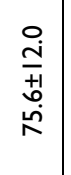 & 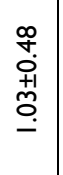 & $\begin{array}{c}\hat{N} \\
0 \\
+1 \\
\\
\end{array}$ & 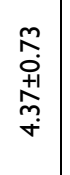 & $\begin{array}{l}\overline{\dot{x}} \\
\overline{+1} \\
\stackrel{\dot{N}}{\partial}\end{array}$ & $\begin{array}{l}0 \\
\dot{0} \\
+1 \\
\dot{+1} \\
\dot{0}\end{array}$ & 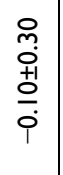 & 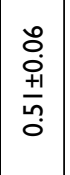 & \\
\hline & 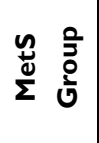 & 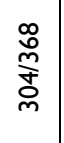 & $\begin{array}{l}\stackrel{\dot{\omega}}{\dot{\omega}} \\
\frac{1}{+1} \\
\dot{\sigma}\end{array}$ & 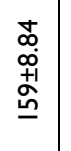 & 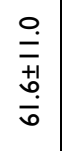 & $\begin{array}{l}\overline{0} \\
\infty \\
\infty \\
\infty \\
\infty \\
\infty \\
\infty\end{array}$ & $\begin{array}{c}\infty \\
\stackrel{\infty}{+} \\
\sim \\
\stackrel{+}{+} \\
\stackrel{\sim}{\sim} \\
\stackrel{\sim}{\sim}\end{array}$ & $\begin{array}{l}\stackrel{0}{\circ} \\
\stackrel{+}{+1} \\
\stackrel{0}{0}\end{array}$ & $\begin{array}{c}\tilde{I} \\
\mathbb{1} \\
+1 \\
\dot{\infty} \\
\dot{\infty}\end{array}$ & 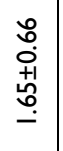 & $\begin{array}{c}\tilde{Z} \\
0 \\
+1 \\
0 \\
0 \\
-1\end{array}$ & $\begin{array}{c}\sigma \\
\dot{\sigma} \\
+1 \\
\stackrel{+1}{\alpha} \\
\dot{+}\end{array}$ & 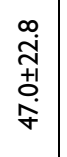 & 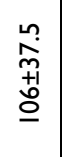 & 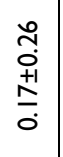 & $\left|\begin{array}{l}n \\
0 \\
0 \\
+1 \\
n \\
n \\
0\end{array}\right|$ & \\
\hline \multirow{4}{*}{ 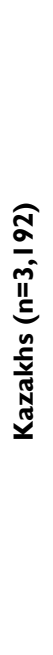 } & 旁 & $\frac{\tilde{\Xi}}{0}$ & $\begin{array}{l}\overline{8} \\
\text { i }\end{array}$ & $\frac{\pi}{\sigma}$ & $\begin{array}{l}\bar{\delta} \\
\dot{0} \\
\mathrm{v}\end{array}$ & $\begin{array}{l}\bar{\delta} \\
\dot{i} \\
\mathrm{v}\end{array}$ & $\begin{array}{l}\bar{\delta} \\
\dot{0} \\
\mathrm{v}\end{array}$ & $\begin{array}{l}\bar{o} \\
\dot{0} \\
\dot{v}\end{array}$ & $\begin{array}{l}\bar{\delta} \\
\dot{\mathrm{v}}\end{array}$ & $\begin{array}{l}\overline{\dot{o}} \\
\dot{\mathrm{v}}\end{array}$ & \begin{tabular}{|l}
$\bar{\delta}$ \\
$\dot{0}$ \\
$\mathrm{v}$
\end{tabular} & $\begin{array}{l}\overline{8} \\
\dot{0} \\
\mathrm{v}\end{array}$ & $\begin{array}{l}\bar{\delta} \\
\dot{i} \\
\mathrm{v}\end{array}$ & $\begin{array}{l}\bar{\delta} \\
\dot{i} \\
\mathrm{v}\end{array}$ & $\begin{array}{l}\overline{\mathrm{o}} \\
\dot{\mathrm{v}} \\
\mathrm{v}\end{array}$ & $\begin{array}{l}\overline{8} \\
\text { iे }\end{array}$ & \\
\hline & $\underset{\mathbb{D}}{\widetilde{J}}$ & 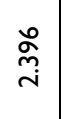 & $\begin{array}{l}\infty \\
\stackrel{\infty}{0} \\
\frac{1}{1}\end{array}$ & $\frac{\infty}{0}$ & $\begin{array}{l}\text { \& } \\
\infty \\
\underset{+}{+} \\
\end{array}$ & 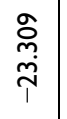 & $\frac{\circ}{\frac{0}{\alpha}}$ & 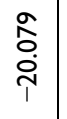 & $\begin{array}{c}\stackrel{2}{\hat{\alpha}} \\
\stackrel{\alpha}{1} \\
1\end{array}$ & 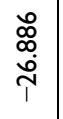 & 疋 & 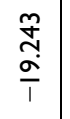 & $\frac{\infty}{\stackrel{\infty}{+}}$ & $\begin{array}{l}\stackrel{8}{\circ} \\
\stackrel{+}{+} \\
\hat{i}\end{array}$ & 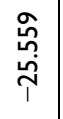 & $\left|\begin{array}{c}\tilde{\tau} \\
\underset{\sim}{\sim} \\
\tilde{i}\end{array}\right|$ & \\
\hline & 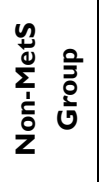 & $\stackrel{\frac{n}{m}}{\stackrel{\equiv}{二}}$ & $\begin{array}{l}\overline{\mathbf{u}} \\
\overline{+1} \\
\dot{+} \\
\dot{\sigma}\end{array}$ & 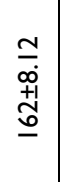 & 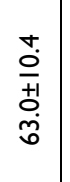 & 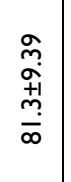 & $\begin{array}{l}\overline{\bar{p}} \\
\dot{p} \\
\dot{p} \\
\dot{\sim}\end{array}$ & 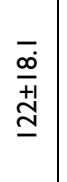 & $\left|\begin{array}{l}\infty \\
0 \\
0 \\
+1 \\
\infty\end{array}\right|$ & 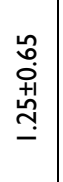 & 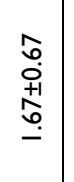 & $\begin{array}{c}\sigma \\
\sigma \\
+1 \\
+1 \\
\infty \\
\dot{\sigma} \\
+\end{array} \mid$ & 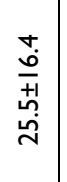 & $\begin{array}{l}\stackrel{0}{\dot{m}} \\
\stackrel{+}{+} \\
\stackrel{+}{*} \\
\end{array}$ & 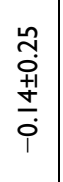 & $\begin{array}{l}0 \\
0 \\
0 \\
0 \\
0 \\
0 \\
0 \\
0\end{array}$ & \\
\hline & 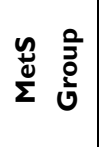 & 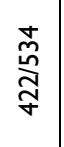 & 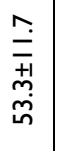 & $\begin{array}{l}\stackrel{R}{0} \\
\infty \\
+1 \\
\text { d } \\
\underline{0}\end{array}$ & $\frac{\stackrel{n}{0}}{\frac{0}{+1}}$ & $\begin{array}{c}\bar{n} \\
+ \\
\tilde{m} \\
\dot{\omega} \\
\infty\end{array} \mid$ & 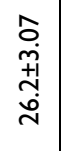 & $\begin{array}{l}\stackrel{a}{0} \\
\stackrel{+}{1} \\
\stackrel{0}{0} \\
\underline{m}\end{array}$ & $\left|\begin{array}{c}\sigma \\
\overline{\bar{亠}} \\
\stackrel{+1}{\alpha}\end{array}\right|$ & 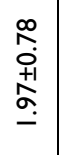 & $\begin{array}{l}\hat{R} \\
0 \\
+1 \\
\hat{1} \\
\underline{n}\end{array} \mid$ & $\begin{array}{l}\mathfrak{d} \\
\dot{i} \\
\tilde{N} \\
\sigma \\
\dot{n}\end{array}$ & 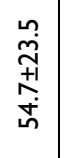 & 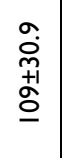 & $\begin{array}{l}\text { 望 } \\
\text { †1 } \\
\text { 응 }\end{array}$ & 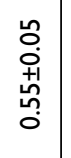 & \\
\hline$\frac{1}{3}$ & & 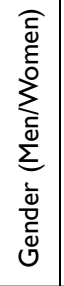 & 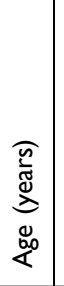 & 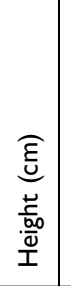 & 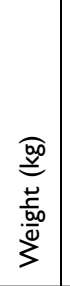 & $\frac{\underset{U}{\tilde{E}}}{\bar{z}}$ & 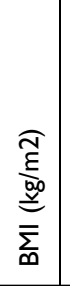 & 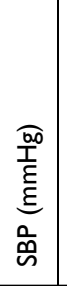 & 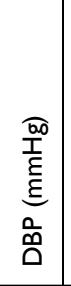 & 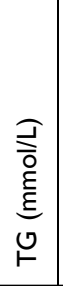 & 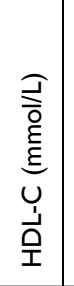 & 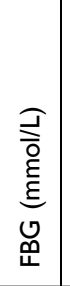 & $\stackrel{q}{s}$ & U & $\frac{0}{<}$ & 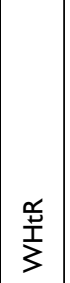 & $\stackrel{*}{*}$ \\
\hline
\end{tabular}



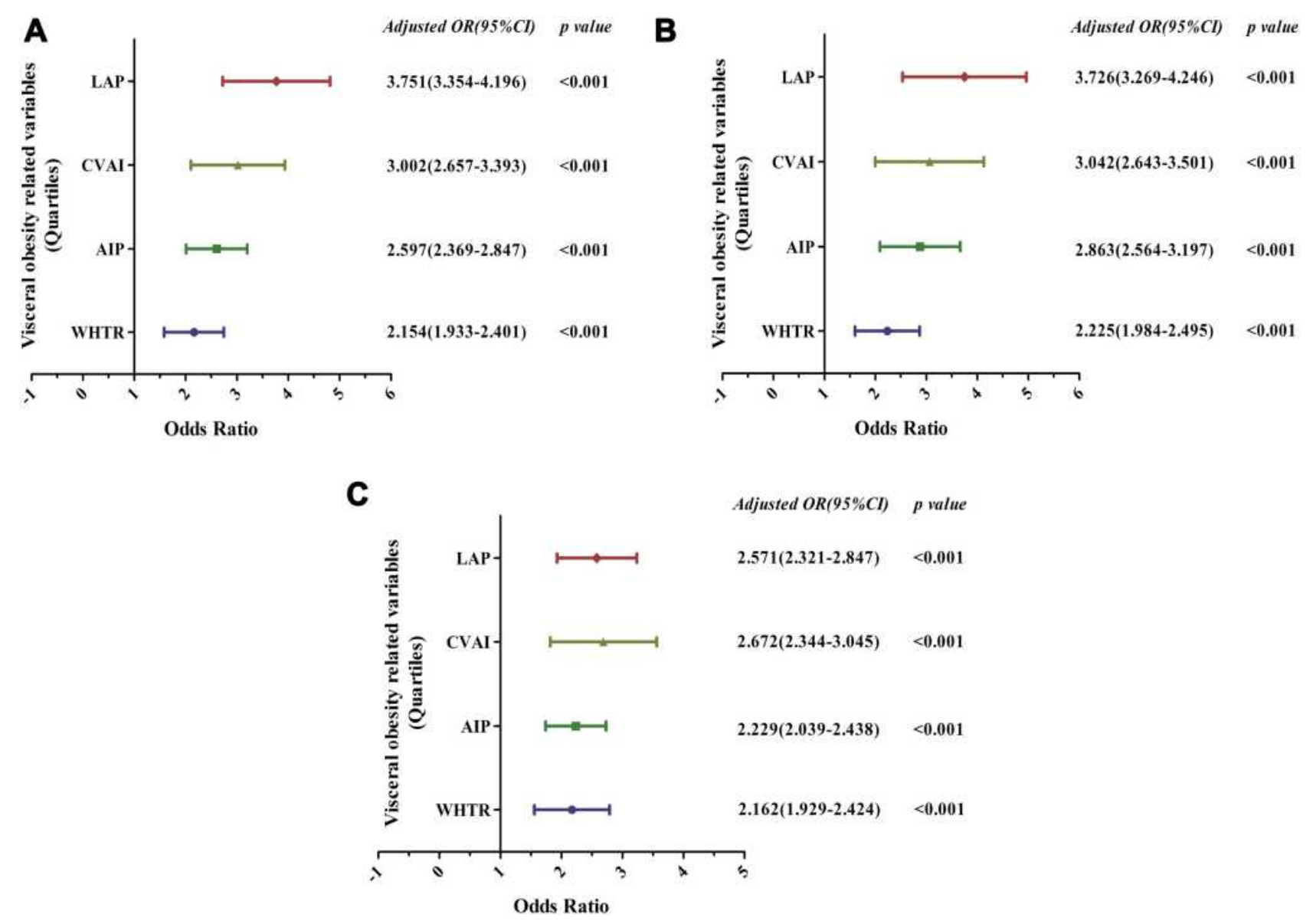

Figure 2 Adjusted odds ratios and $95 \% \mathrm{Cl}$ of MetS according to levels of visceral obesity-related indices for Kazakhs, Uyghurs and Hans, respectively (A) - Kazakhs, (B) Uyghurs and (C) - Hans).

Note: Data was adjusted for age, sex, smoking and drinking habits, occupation, marital status, education and BMI.

Abbreviations: $\mathrm{Cl}$, confidence interval; LAP, lipid accumulation product; CVAI, Chinese visceral adiposity index; AIP, atherogenic index of plasma; WHtR, waist-to-height ratio.

and effective tool for screening MetS among different ethnicities, and will lay a foundation for the screening and diagnosis of other metabolic diseases.

There have been many studies focused on the prediction of MetS. ${ }^{28-30}$ However, it is clear that there are and will continue to be differences in body fat distribution among ethnic groups. For example, thresholds of BMI, WC, and WHtR have been recommended in several different ethnic groups and populations in World Health Organization guidelines. ${ }^{4,31}$ Despite these improved guidelines, one key question is whether the same criteria should be applied to someone of a particular country regardless of their subgroup ethnic. Various studies have additionally documented that the Chinese population also possesses subgroup ethnic variations. These need to be validated for various communities and ethnic groups. ${ }^{32,33}$ However, only little data on low-income areas in China are available due to reasons such as low priority of public health, poor medical services, transportation inconveniences, and language communication barriers in these areas. These gaps result in limitations in policy-making for public health in remote rural areas.

Evidence has shown that visceral obesity plays a central role in MetS and is an independent marker of cardiovascular risk, morbidity, and mortality. ${ }^{34,35}$ Since routine access to CT or MRI might not be feasible for many clinicians, these surrogate indices of visceral obesity are vital in screening for MetS. The present study revealed that there were significant relations between LAP, CVAI, AIP, as well as WHtR and MetS in all the three ethnicities after adjusting for confounding factors. Furthermore, the ORs of LAP were highest in both Kazakhs and Uyghurs, while CVAI indicated the highest OR in Hans when compared to the other three indices. This is explained by the 
A

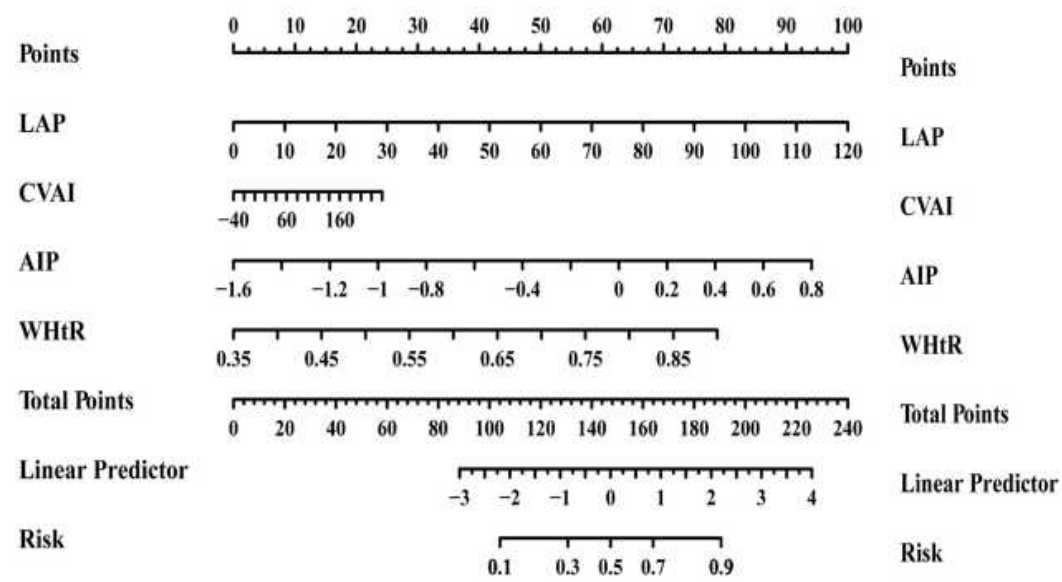
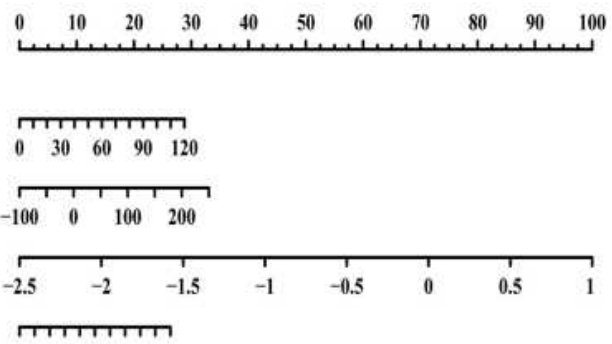

$\begin{array}{lll}0.35 & 0.55 & 0.75\end{array}$

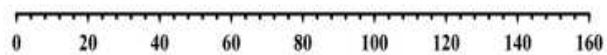

$\begin{array}{ccccccc}-3 & -1.5 & 0 & 1 & 2 & 3 & 4\end{array}$

$\begin{array}{llll}0.1 & 0.4 & 0.7 & 0.9\end{array}$

\section{C}

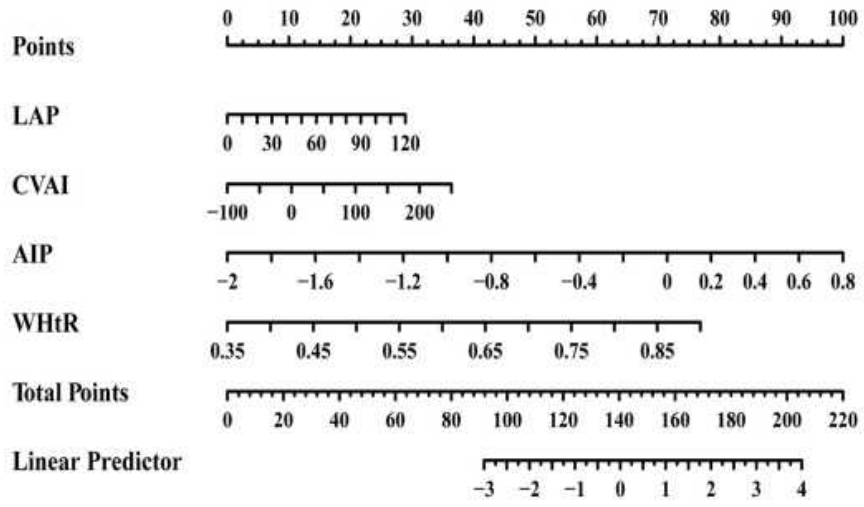

Risk

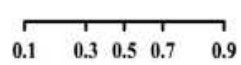

Figure 3 Nomogram to estimate the risk of MetS for Kazakhs, Uyghurs and Hans (A) - Kazakhs, (B) - Uyghurs and (C) - Hans).

Note: To use the nomogram, determine the position of each index on the respective axis, draw a vertical line to the points bar to obtain the points of each index, add all the points, and then draw a vertical line from the total points bar to the risk axis to determine the risk of MetS.

Abbreviations: LAP, lipid accumulation product; CVAI, Chinese visceral adiposity index; AIP, atherogenic index of plasma; WHtR, waist-to-height ratio.

fact that the CVAI is developed from Chinese Han adults, which may be more practical for the Chinese Han population. However, the risk for MetS when WHtR increased was not as significant compared to the other indicators in all Kazakhs, Uyghurs, and Hans. This may be due to the fact that WHtR can not accurately identify the origin of adipose tissue in the abdominal region. ${ }^{10}$

According to Hosmer and Lemeshow's criteria, ${ }^{25}$ the present study found that LAP, CVAI, AIP, and WHtR were useful indices in screening for MetS (the AUC values were all greater than 0.7 ). This is in accord with the fact that visceral obesity-related indices might provide a broader evaluation of metabolic risk related to metabolic dysfunction and fat distribution. ${ }^{36}$ The LAP was an "excellent" index (AUC $=0.853$ for Kazakhs, $\mathrm{AUC}=0.851$ for Uyghurs, and $\mathrm{AUC}=0.798$ for Hans, almost close to 0.8 ). In all of Kazakhs, Uyghurs, and Hans, LAP was able to most accurately identify individuals with or without MetS compared to the other three indices. Consistent with previous studies, ${ }^{14,37}$ the findings of this study further highlighted the value of LAP in screening MetS across different diseases and ethnic groups. The combined presence of increased WC and TG levels has been associated with a high probability (around $80 \%$ ) of increased visceral adipose tissue levels. ${ }^{38}$ These findings have been confirmed in other studies, deeming the so-called "hypertriglyceridemic waist" as a simple tool to screen for the presence of excess visceral adipose tissue. ${ }^{39}$ However, visceral obesity is a continuous process rather than a dichotomous one, while both $\mathrm{WC}$ and $\mathrm{TG}$ are single continuous variables are 


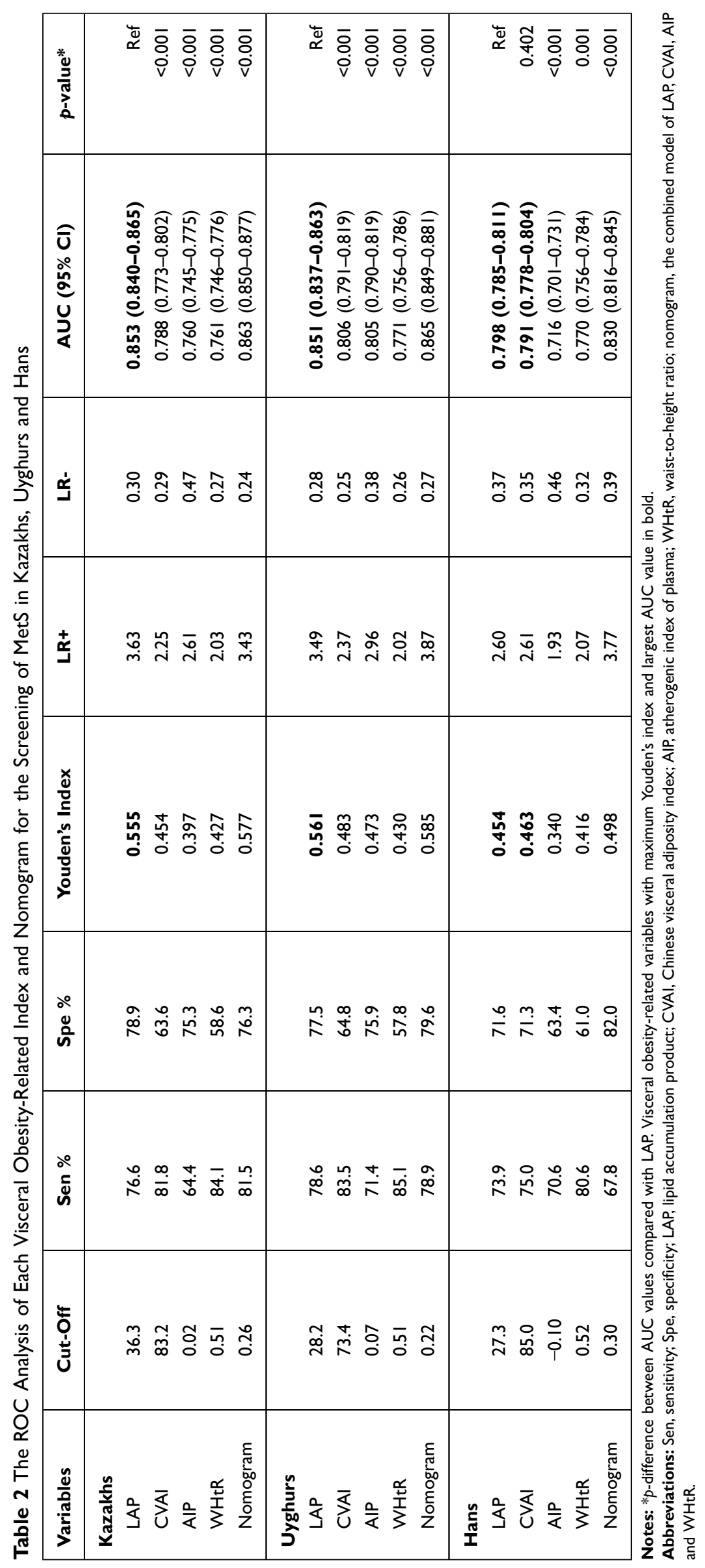



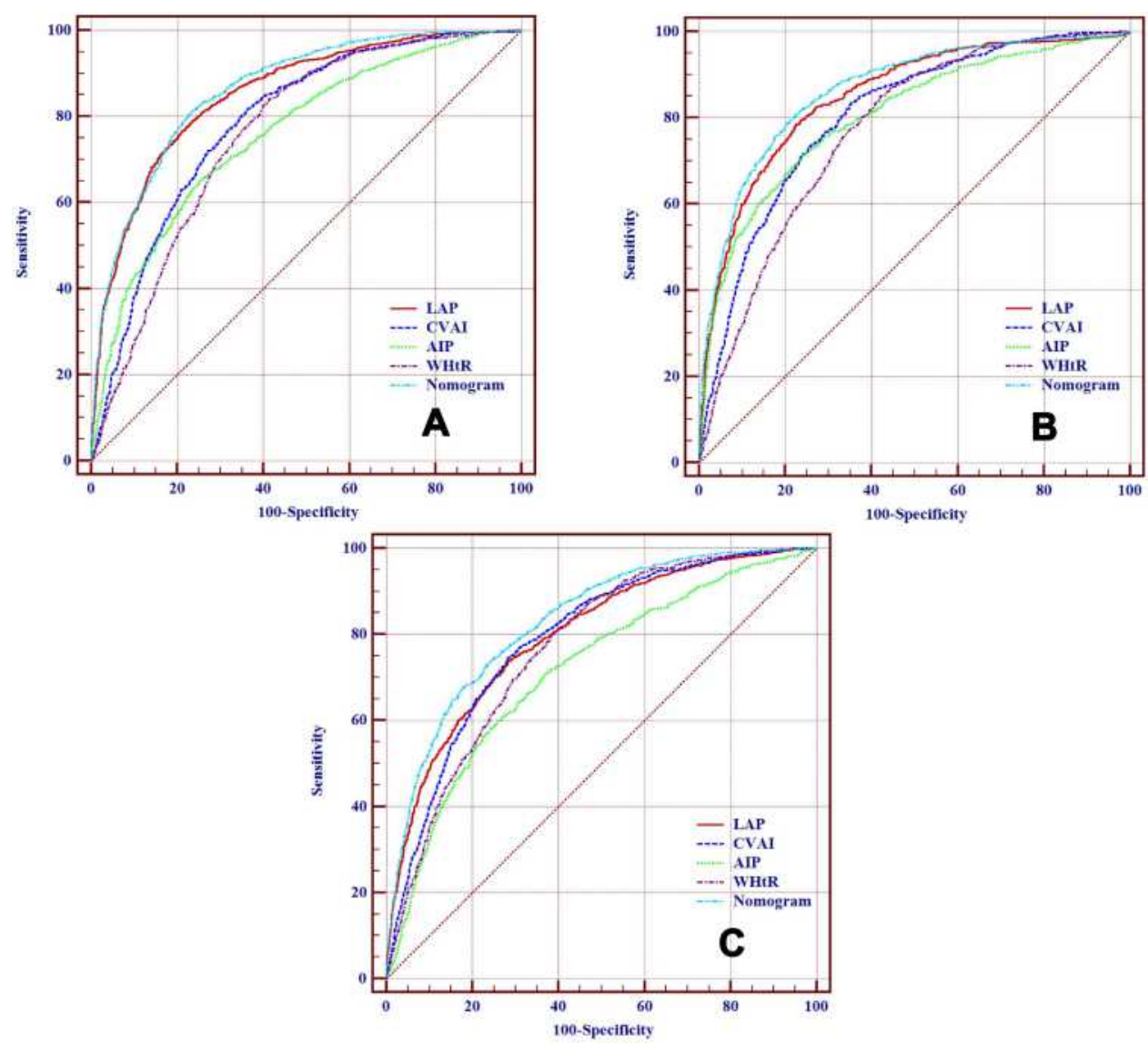

Figure 4 ROC curves of each visceral obesity-related index for the screening of MetS in Kazakhs, Uyghurs and Hans according to the JIS criteria (A)- Kazakhs, (B)- Uyghurs and (C)- Hans).

Abbreviations: LAP, lipid accumulation product; CVAI, Chinese visceral adiposity index; AIP, atherogenic index of plasma; WHtR, waist-to-height ratio; nomogram, the combined model of LAP, CVAI, AIP and $\mathrm{WH} t \mathrm{R}$.

associated with metabolic risk. ${ }^{11}$ Thus, LAP is suggested to expand on the concept of the hypertriglyceridemic waist by providing a continuous risk marker that better reflects the excess lipid accumulation in visceral obesity and its related CVD risk. ${ }^{11}$ This related risk indicates the underlying continuous process which follows the over-accumulation of visceral adipose tissue, gradually promoting metabolic dysregulation, atherosclerosis, and low-grade inflammation. ${ }^{40}$

CVAI was reported to be a reliable index in predicting visceral adipose area and MetS in Chinese adults. The findings of this study were consistent with previous data regarding CVAI as a better marker of MetS in Hans. ${ }^{16}$ These results suggest that CVAI may be better than other visceral obesity indices at identifying MetS in the Chinese Han population. Interestingly, CVAI, which is developed on the basis of age, BMI, WC, TG, and HDL-C, ${ }^{16}$ includes more metabolic risk factors than those in the LAP, ${ }^{11}$ but the AUC values of CVAI were poorer than that of LAP in both Kazakhs and Uyghurs. Even in Hans, the AUC values of VAI and LAP did not differ statistically. It was further confirmed that LAP may be more accurate in screening for MetS in different ethnic groups.

AIP was another strong marker for predicting the risk of atherosclerosis and $\mathrm{CVD}^{24,41}$ and was more convenient and economical compared with B-mode ultrasound or CT. To date, few studies have evaluated the value of AIP for the screening of MetS. In agreement with a recent study, ${ }^{42}$ the present study showed that AIP was an "excellent" indicator for Uyghurs (AUC $=0.805)$ and an "acceptable" indicator for both Kazakhs and Hans $(\mathrm{AUC}=0.760$ and 0.716). However, AIP performed lower than LAP and CVAI, which may be due to the fact that AIP does not engage WC. Studies have shown that the combination of WC and AIP may increase their specificity and sensitivity for detection of visceral obesity in clinical practice. ${ }^{18}$ Thus, further studies are required to fully elucidate the screening value of AIP for MetS. 
Furthermore, the values of these indices of interest for the screening of MetS in Hans were generally lower than that in Kazakhs or Uyghurs. Although the exact mechanism of these disparities remains to be elucidated, it could be, at least in part, due to the remarkable differences in adipose tissue distribution between Kazakhs, Uyghurs, and Hans. Kazakhs and Uyghurs may be more prone to have visceral adipose accumulation than that in Hans. In addition, this study also evaluated the value of combining the use of visceral obesity indices in screening for MetS, with results showing that the AUC values of combined screening for each ethnic group were higher than those when using the indices in isolation. However, compared with LAP, the improved value of combined screening was not significant (AUC: 0.863 vs 0.853 in Kazakhs, 0.865 vs 0.851 in Uyghurs, and 0.830 vs 0.798 in Hans, respectively).

Taken together, LAP may be of clinical applicability in detecting MetS in various ethnic groups. Until now, there is no official cut-off for LAP in screening for MetS. In this regard, the present study obtained optimal cut-offs of 36.3 for Kazakhs, 28.2 for Uyghurs, and 27.3 for Hans, respectively. These results differed from other similar studies, such as a study in Brazilian adults (34.2) ${ }^{14}$ and the Iranian population (49.7 for women and 39.9 for men), ${ }^{37}$ which may have been due to ethnic differences in environmental exposure and genetics. Moreover, the different MetS definitions may also contribute to the discrepancy in the optimal cut-off points. Although the sensitivity of LAP for the screening of MetS in each ethnic group was lower than that of CVAI and WHtR, the cut-off points of LAP indicated sensitivity and specificity values between $70 \%$ and $80 \%$ for all Kazakhs, Uyghurs, and Hans, thus moderately minimizing false-positive and false-negative cases.

Several limitations of the present study should be considered. First, its cross-sectional design limits the researchers from making causal inferences. Second, only four widelyused visceral obesity indices were analyzed in the study. There may be other novel indicators, which this study did not consider. Third, the MetS groups were not subdivided by sex. Therefore, further studies with prospective designs, more accurate indicators, and detailed information collection are needed to assess the screening value of visceral obesity-related indices in identifying individuals at high risk of MetS.

In conclusion, the present study investigated the screening ability of visceral obesity-related indices, including LAP, CVAI, AIP, and WHtR, in identifying MetS. LAP was the best index in identifying risk for MetS in all Kazakhs, Uyghurs, and Hans, with the optimal cut-offs being 36.3, 28.2 , and 27.3 respectively. The screening ability of CVAI for MetS was similar to LAP in Hans, with the optimal cut-off being 85.0. LAP may be a complementary indicator for screening for MetS in various ethnic groups.

\section{Abbreviations}

MetS, metabolic syndrome; T2DM, type 2 diabetes; CVD, cardiovascular disease; JIS, Joint Interim Statement; ROC, receiver operating characteristic curve; AUC, area under the ROC curve; LAP, Lipid accumulation product; CVAI, Chinese visceral adiposity index; AIP, atherogenic index of plasma; WHtR: waist-to-height ratio; WC, waist circumference; BMI, body mass index; IDF, International Diabetes Federation; HDL-C, high-density lipoprotein cholesterol; TG, triglycerides; SBP, systolic blood pressure; DBP, diastolic blood pressure; FBG, fasting blood glucose; MRI, magnetic resonance imaging; CT, computed tomography; OR, odds ratio; CI, confidence interval; Sen, sensitivity; Spe, specificity.

\section{Data Sharing Statement}

The data used in this study can be obtained from the corresponding authors according to reasonable requirements.

\section{Ethics Approval and Informed Consent}

The study was conducted according to the guidelines of the Declaration of Helsinki, and approved by the Ethics Committee of name of the First Affiliated Hospital of Shihezi University School of Medicine (IERB No. SHZ2010LL01). Informed consent was obtained from all subjects involved in the study. Participation was strictly voluntary, and anonymity was guaranteed.

\section{Acknowledgments}

The authors sincerely thank the Health Bureaus of study region, the 51st Regiment Hospital and their staff for their on-site assistance. The authors are also grateful to the clinical laboratory of the First Affiliated Hospital of Shihezi University School of Medicine for the testing work.

\section{Author Contributions}

All authors made a significant contribution to the work reported, whether that is in the conception, study design, execution, acquisition of data, analysis and interpretation, or in all these areas; took part in drafting, revising or critically reviewing the article; gave final approval of the version to be published; have agreed on the journal to which the article has been submitted; and agree to be 
accountable for all aspects of the work. Shuxia Guo and Rulin Ma were co-corresponding authors in the present study. Xianghui Zhang and Yusong Ding contributed equally to this work.

\section{Funding}

This research was supported by the Youth Innovation Training Talent Project of Shihezi University (No. CXPY201908) and National Natural Science Foundation of China (NSFC, No. 81560551).

\section{Disclosure}

The authors declare no conflict of interests for this work.

\section{References}

1. Saklayen MG. The global epidemic of the metabolic syndrome. Curr Hypertens Rep. 2018;20(2):12. doi:10.1007/s11906-018-0812-z

2. Isomaa B, Almgren $\mathrm{P}$, Tuomi $\mathrm{T}$, et al. Cardiovascular morbidity and mortality associated with the metabolic syndrome. Diabetes Care 2001;24(4):683-689. doi:10.2337/diacare.24.4.683

3. Alberti KGMM, Zimmet P, Shaw J; Group IETFC. The metabolic syndrome - a new worldwide definition. Lancet. 2005;366 (9491):1059-1062. doi:10.1016/S0140-6736(05)67402-8

4. Alberti K, Eckel RH, Grundy SM, et al. Harmonizing the metabolic syndrome: a joint interim statement of the international diabetes federation task force on epidemiology and prevention; national heart, lung, and blood institute; American heart association; world heart federation; international atherosclerosis society; and international association for the study of obesity. Circulation. 2009;120 (16):1640-1645. doi:10.1161/CIRCULATIONAHA.109.192644

5. Heart AHA. National. diagnosis and management of the metabolic syndrome: an American heart association/national heart, lung, and blood institute scientific statement. Curr Opin Cardiol. 2005;21 (1):1-6.

6. Tchernof A, Després JP. Pathophysiology of human visceral obesity: an update. Physiol Rev. 2013;93(1):359-404. doi:10.1152/ physrev.00033.2011

7. Moran RA, García-Rayado G, de la Iglesia-garcía D, et al. Influence of age, body mass index and comorbidity on major outcomes in acute pancreatitis, a prospective nation-wide multicentre study. United European Gastroenterol J. 2018;6(10):1508-1518. doi:10.1177/ 2050640618798155

8. Nazare J-A, Smith JD, Borel A-L, et al. Ethnic influences on the relations between abdominal subcutaneous and visceral adiposity, liver fat, and cardiometabolic risk profile: the international study of prediction of intra-abdominal adiposity and its relationship with cardiometabolic risk/intra-abdominal adiposity. Am J Clin Nutr. 2012;96(4):714-726. doi:10.3945/ajcn.112.035758

9. Neeland IJ, McGuire DK, Eliasson B, et al. Comparison of adipose distribution indices with gold standard body composition assessments in the EMPA-REG H2H SU Trial: a body composition sub-study. Diabetes Ther. 2015;6(4):635-642. doi:10.1007/s13300-015-0146-7

10. Neeland IJ, Ross R, Després JP, et al. Visceral and ectopic fat, atherosclerosis, and cardiometabolic disease: a position statement. Lancet Diabetes Endocrinol. 2019;7(9):715-725. doi:10.1016/ S2213-8587(19)30084-1

11. Kahn HS. The" lipid accumulation product" performs better than the body mass index for recognizing cardiovascular risk: a populationbased comparison. BMC Cardiovasc Disord. 2005;5(26). doi:10.1186/1471-2261-5-26
12. Mazidi M, Kengne AP, Katsiki N, Mikhailidis DP, Banach M. Lipid accumulation product and triglycerides/glucose index are useful predictors of insulin resistance. J Diabetes Complications. 2017;32 (3):266-270. doi:10.1016/j.jdiacomp.2017.10.007

13. Dai H, Wang W, Chen R, Chen Z, Lu Y, Yuan H. Lipid accumulation product is a powerful tool to predict non-alcoholic fatty liver disease in Chinese adults. Nutr Metab. 2017;14(1):49. doi:10.1186/s12986-0170206-2

14. Nascimento-Ferreira MV, Rendo-Urteaga T, Vilanova-Campelo RC, et al. The lipid accumulation product is a powerful tool to predict metabolic syndrome in undiagnosed Brazilian adults. Clin Nutr. 2017;36(6):1693-1700. doi:10.1016/j.clnu.2016.12.020

15. Barreira TV, Broyles ST, Gupta AK, Katzmarzyk PT. Relationship of anthropometric indices to abdominal and total body fat in youth: sex and race differences. Obesity (Silver Spring). 2014;22(5):1345-1350. doi:10.1002/oby.20714

16. Xia MF, Chen Y, Lin HD, et al. A indicator of visceral adipose dysfunction to evaluate metabolic health in adult Chinese. Sci Rep. 2016;6(1):38214. doi:10.1038/srep38214

17. Dobiásová M, Frohlich J. The plasma parameter log (TG/HDL-C) as an atherogenic index: correlation with lipoprotein particle size and esterification rate in apoB-lipoprotein-depleted plasma (FER(HDL)). Clin Biochem. 2001;34(7):583-588. doi:10.1016/S0009-9120(01)00263-6

18. Shen SW, Lu Y, Li F, et al. Atherogenic index of plasma is an effective index for estimating abdominal obesity. Lipids Health Dis. 2018;17(1):11. doi:10.1186/s12944-018-0656-1

19. Bora K, Pathak MS, Borah P, Hussain MI, Das D. Association of the apolipoprotein A-I gene polymorphisms with cardiovascular disease risk factors and atherogenic indices in patients from Assam, Northeast India. Balkan J Med Genet. 2017;20(1):59-70. doi:10.1515/bjmg-2017-0002

20. Yang SH, Du Y, Li XL, et al. Triglyceride to high-density lipoprotein cholesterol ratio and cardiovascular events in diabetics with coronary artery disease. Am J Med Sci. 2017;354(2):117-124. doi:10.1016/j. amjms.2017.03.032

21. Deurenberg P, Yap M, van Staveren WA. Body mass index and percent body fat: a meta analysis among different ethnic groups. Int $J$ Obes Relat Metab Disord. 1998;22(12):1164-1171. doi:10.1038/sj.ijo.0800741

22. Guo H, Gao X, Ma R, et al. Prevalence of metabolic syndrome and its associated factors among multi-ethnic adults in rural areas in Xinjiang, China. Sci Rep. 2017;7(1):17643. doi:10.1038/s41598017-17870-5

23. Gomez F, Corchuelo J, Curcio CL, Calzada MT, Mendez F. SABE colombia: survey on health, well-being, and aging in Colombia-study design and protocol. Curr Gerontol Geriatr Res. 2016;2016:7910205. doi:10.1155/2016/7910205

24. Fernández-Macías JC, Ochoa-Martínez AC, Varela-Silva JA, PérezMaldonado IN. Atherogenic index of plasma: novel predictive biomarker for cardiovascular illnesses. Arch Med Res. 2019;50(5):285294. doi:10.1016/j.arcmed.2019.08.009

25. Hosmer DW, Lemeshow S. Applied Logistic Regression. 2nd ed. NY: John Wiley \& Sons; 2000. doi:10.1002/0471722146.ch4

26. Yang H, Lu K, Lyu X, Hu F. Two-way partial AUC and its properties. Stat Methods Med Res. 2019;28(1):184-195. doi:10.1177/ 0962280217718866

27. DeLong ER, DeLong DM, Clarke-Pearson DL. Comparing the areas under two or more correlated receiver operating characteristic curves: a nonparametric approach. Biometrics. 1988;44(3):837-845. doi:10.2307/ 2531595

28. Ena A, Aoa B, Aa C, et al. Anthropometric parameter that best predict metabolic syndrome in South west Nigeria. Diabetes Metab Syndr. 2019;13(1):48-54. doi:10.1016/j.dsx.2018.08.009

29. Wu L, Zhu W, Qiao Q, Huang L, Li Y, Chen L. Novel and traditional anthropometric indices for identifying metabolic syndrome in nonoverweight/obese adults. Nutr Metab. 2021;18(1):3. doi:10.1186/ s12986-020-00536-x 
30. Banik SD, Pacheco-Pantoja E, Lugo R, Gómez-de-regil L, Peninsula Y. Evaluation of anthropometric indices and lipid parameters to predict metabolic syndrome among adults in Mexico. Diabetes Metab Syndr Obes. 2021.

31. Semlitsch T, Stigler FL, Jeitler K, Horvath K, Siebenhofer A. Management of overweight and obesity in primary care-A systematic overview of international evidence-based guidelines. Obes Rev. 2019;20(9):1218-1230. doi:10.1111/obr.12889

32. Hui-Fang L, Cai L, Wang XM, Golden AR. Ethnic disparities in prevalence and clustering of cardiovascular disease risk factors in rural Southwest China. BMC Cardiovasc Disord. 2019;19(1):200. doi:10.1186/s12872-019-1185-1

33. Gazzola K, Reeskamp L, Bert-Jan VDB. Ethnicity, lipids and cardiovascular disease. Curr Opin Lipidol. 2017;28(3):1. doi:10.1097/ MOL.0000000000000412

34. Wang T, Ma X, Peng D, et al. Effects of obesity related genetic variations on visceral and subcutaneous fat distribution in a Chinese population. Sci Rep. 2016;6(1):20691. doi:10.1038/srep20691

35. Hiuge-Shimizu A, Kishida K, Funahashi T, et al. Absolute value of visceral fat area measured on computed tomography scans and obesity-related cardiovascular risk factors in large-scale Japanese general population (the VACATION-J study). Ann Med. 2012;44(1):82-92. doi:10.3109/07853890.2010.526138

36. Ramírez-Vélez R, Pérez-Sousa M, González-Ruíz K, et al. Obesityand lipid-related parameters in the identification of older adults with a high risk of prediabetes according to the American diabetes association: an analysis of the 2015 health, well-being, and aging study. Nutrients. 2019;11(11):2654. doi:10.3390/nu11112654
37. Motamed N, Razmjou S, Hemmasi G, Maadi M, Zamani F. Lipid accumulation product and metabolic syndrome: a population-based study in northern Iran, Amol. J Endocrinol Invest. 2016;39(4):375382. doi:10.1007/s40618-015-0369-5

38. Lemieux I, Pascot A, Couillard C, et al. Hypertriglyceridemic waist: a marker of the atherogenic metabolic triad (hyperinsulinemia; hyperapolipoprotein B; small, dense LDL) in men? Circulation. 2000;102 (2):179-184. doi:10.1161/01.CIR.102.2.179

39. LeBlanc S, Coulombe F, Bertrand OF, Bibeau K, Larose E. Hypertriglyceridemic waist: a simple marker of high-risk atherosclerosis features associated with excess visceral adiposity/ectopic fat. $J$ Am Heart Assoc. 2018;7(8):e008139. doi:10.1161/ JAHA.117.008139

40. Kyrou I, Panagiotakos DB, Kouli GM, et al. Lipid accumulation product in relation to 10-year cardiovascular disease incidence in Caucasian adults: the ATTICA study. Atherosclerosis. 2018;279:1016. doi:10.1016/j.atherosclerosis.2018.10.015

41. Wu TT, Gao Y, Zheng YY, Ma YT, Xie X. Atherogenic index of plasma (AIP): a novel predictive indicator for the coronary artery disease in postmenopausal women. Lipids Health Dis. 2018;17 (1):197. doi:10.1186/s12944-018-0828-z

42. Zhang X, Zhang X, Li X, Feng J, Chen X. Association of metabolic syndrome with atherogenic index of plasma in an urban Chinese population: a 15-year prospective study. Nutr Metab Cardiovasc Dis. 2019;29(11):1214-1219. doi:10.1016/j.numecd.2019.07.006

\section{Publish your work in this journal}

Diabetes, Metabolic Syndrome and Obesity: Targets and Therapy is an international, peer-reviewed open-access journal committed to the rapid publication of the latest laboratory and clinical findings in the fields of diabetes, metabolic syndrome and obesity research. Original research, review, case reports, hypothesis formation, expert opinion and commentaries are all considered for publication. The manuscript management system is completely online and includes a very quick and fair peer-review system, which is all easy to use. Visit http://www.dovepress.com/testimonials.php to read real quotes from published authors.

Submit your manuscript here: https://www.dovepress.com/diabetes-metabolic-syndrome-and-obesity-targets-and-therapy-journal 